\title{
Descripción clínica y neurofisiológica de pacientes con el síndrome POEMS
}

\author{
Luis Lee-Chen, Ricardo Williams-de-Roux, Erwin Chiquete*, José Jesús Aceves-Buendía, \\ Eduardo Ruiz-Ruiz, Jennefer Portillo-Valle, Tatiana Bliskunova, Elizabeth Rodríguez-Perea, \\ Liz Toapanta-Yanchapaxi, Guillermo García-Ramos, Carlos Cantú-Brito y Bruno Estañol \\ Departamento de Neurología y Psiquiatría, Instituto Nacional de Ciencias Médicas y Nutrición Salvador Zubirán, Ciudad de México, México
}

\section{Resumen}

Introducción: El síndrome POEMS (polyneuropathy, organomegaly, endocrinopathy, monoclonal protein, skin changes) es una gammapatía monoclonal con polineuropatía como criterio obligatorio. Objetivo: Describir las potenciales asociaciones entre la expresión clínica y los patrones de electrodiagnóstico en el síndrome POEMS. Método: Estudio observacional, retrospectivo y transversal de los casos atendidos en un centro de referencia con diagnóstico de síndrome de POEMS de 2009 a 2019. Resultados: Se analizó a 11 pacientes (8 hombres). La mediana de la edad al diagnóstico fue de 40 años (rango: 31-51, media: $37.19 \pm 15.67$ años). La latencia media al diagnóstico fue de $9.7 \pm 8.37$ meses. En todos la manifestación clínica inicial fue la polineuropatía. La mayoría de los pacientes presentaron un patrón axonal $(n=5)$, seguido del desmielinizante $(n=4)$ y mixto $(n=2)$. La gammapatía monoclonal se observó en todos ( 6 casos $\lambda$ y $5 \mathrm{\kappa}$, inmunoglobulina [lg] G: 72\%, IgA: 18\%, IgM: 9\%). El Medical Research Council sum score fue menor en el patrón axonal (medianas: 37.00 vs. 45.5, $p=0.024)$. No se presentaron diferencias en la afección sistémica entre patrones electrofisiológicos. Conclusión: Es poco probable que los patrones electrofisiológicos tengan una correspondencia clínica extraneurológica clara, sin embargo, esto deberá probarse de forma definitiva con un mayor tamaño de muestra.

PALABRAS CLAVE: Axonal. Desmielinizante. POEMS. Polineuropatía. Síndrome.

\section{Clinical and neurophysiological description of patients with POEMS syndrome}

\begin{abstract}
Introduction: POEMS syndrome (polyneuropathy, organomegaly, endocrinopathy, monoclonal protein, skin changes) is a monoclonal gammopathy with polyneuropathy as a mandatory criterion. Objective: To describe potential associations between clinical expression and electrodiagnostic patterns in POEMS syndrome. Method: Observational, retrospective, and cross-sectional study of cases cared in a referral center diagnosed with POEMS syndrome from 2009-2019. Results: Eleven cases (8 men) were analyzed. Median age at diagnosis was 40 years (range: 31-51, mean: $37.19 \pm 15.67$ years). Mean latency at diagnosis was 9.7 \pm 8.37 months. In all subjects, initial clinical manifestation was polyneuropathy. Most patients had an axonal pattern $(n=5)$, followed by demyelinating $(n=4)$, and mixed $(n=2)$. Monoclonal gammopathy was observed in all patients $(6 \lambda$, and $5 \kappa$ cases, immunoglobulin [Ig] G: 72\%, IgA: 18\%, IgM: 9\%). Medical Research Council sum score was lower in the axonal pattern (median: 37.00 , vs. $45.5, p=0.024$ ). There were no differences in the systemic involvement between electrophysiological patterns. Conclusion: Electrophysiological patterns are unlikely to have a clear extra-neurological clinical correspondence; however this will need to be definitively proven with a larger sample size.
\end{abstract}

KEY WORDS: Axonal. Desmyelinating. POEMS. Polineuropathy. Syndrome.

Correspondencia:

${ }^{\star}$ Erwin Chiquete

E-mail: erwin.chiquetea@incmnsz.mx

0016-3813/๑ 2021 Academia Nacional de Medicina de México, A.C. Publicado por Permanyer. Este es un artículo open access bajo la licencia CC BY-NC-ND (http://creativecommons.org/licenses/by-nc-nd/4.0/).
Fecha de recepción: 14-01-2021

Fecha de aceptaciçon: 08-03-2021

Gac Med Mex. 2021;157:484-490

Disponible en PubMed

www.gacetamedicademexico.com 


\section{Introducción}

El síndrome POEMS (polyneuropathy, organomegaly, endocrinopathy, monoclonal protein, skin changes), o enfermedad de Crow-Fukase, es un desorden de células plasmáticas con implicación sistémica cuyo acrónimo describe las características del síndrome: polineuropatía, organomegalia, endocrinopatía, proteína $\mathrm{M}$ y cambios en la piel, aunque no todos los sistemas afectados están en relación con el acrónimo ${ }^{1,2}$. No existe un examen diagnóstico que sea específico, se requiere la presencia de tres criterios mayores: polineuropatía, gammapatía monoclinal eminentemente $\lambda$, lesiones óseas, elevación del factor de crecimiento del endotelio vascular (VEGF) y/o enfermedad de Castleman, con al menos uno de seis criterios menores $^{3}$ (Tabla 1). Existe un ligero predominio en hombres, con una relación de 1.4:1, con una mediana de la edad al diagnóstico de entre 45-50 años, según la serie ${ }^{4-6}$. Se estima que la prevalencia es de aproximadamente 0.3 por 100,000 habitantes 7,8 . Al ser una enfermedad rara, suele ser poco incluida en el diagnóstico diferencial de una neuropatía desmielinizante, lo que conduce a errores de diagnóstico y tratamiento ${ }^{9}$.

La neuropatía suele ser una de las manifestaciones iniciales que se presenta con afección sensitivomotora, de predominio distal y simétrica, pero que llega a afectar las cuatro extremidades ${ }^{10,11}$. Electrofisiológicamente la neuropatía es de predominio desmielinizante, particularmente de los segmentos intermedios más que en los distales y daño axonal subsecuente de predominio distal, tanto en nervios motores como sensitivos ${ }^{11,12}$. Es posible que se encuentre un patrón de afectación mixto al momento del diagnóstico ${ }^{13}$.

EI POEMS es una entidad tratable, pudiéndose evitar que progrese hasta la pérdida de la deambulación en el $75 \%$ de los casos con tratamiento oportuno ${ }^{14,15}$. EI VEGF tiene un buen desempeño de diagnóstico en el síndrome POEMS, además de ser una pauta para evaluar la respuesta terapéutica. A diferencia de lo que ocurre en otras neuropatías crónico-degenerativas $^{16,17}$, con la excepción del VEGF, las características de imagen o de laboratorio tienen un papel mucho menor en el monitoreo clínico de la evolución y de la respuesta al tratamiento ${ }^{1,2,15}$, aunque es probable que las características electrofisiológicas puedan cumplir con este papel, pero ello requiere de más evidencia clínica ${ }^{11,13}$.

La investigación clínica sobre la neuropatía del síndrome POEMS es también escasa ${ }^{11-19}$. Esta se ha
Tabla 1. Actualización 2019 sobre los criterios diagnósticos del síndrome POEMS

\begin{tabular}{|c|c|}
\hline $\begin{array}{l}\text { Categoría } \\
\text { diagnóstica }\end{array}$ & Criterios \\
\hline \multirow{2}{*}{$\begin{array}{l}\text { Criterios mayores } \\
\text { mandatorios } \\
\text { (ambos requeridos) }\end{array}$} & 1. Polineuropatía \\
\hline & $\begin{array}{l}\text { 2. Trastorno monoclonal de células } \\
\text { plasmáticas (en su mayoría } \lambda \text { ) }\end{array}$ \\
\hline \multirow{3}{*}{$\begin{array}{l}\text { Otros criterios } \\
\text { mayores } \\
\text { (uno requerido) }\end{array}$} & 3. Enfermedad de Castleman \\
\hline & 4. Lesiones osteoescleróticas \\
\hline & 5. Elevación de VEGF \\
\hline \multirow[t]{6}{*}{$\begin{array}{l}\text { Criterios menores } \\
\text { (uno requerido) }\end{array}$} & $\begin{array}{l}\text { 6. Organomegalia (esplenomegalia, } \\
\text { hepatomegalia o linfadenopatía) }\end{array}$ \\
\hline & $\begin{array}{l}\text { 7. Sobrecarga de volumen extravascular } \\
\text { (edema, derrame pleural, ascitis) }\end{array}$ \\
\hline & $\begin{array}{l}\text { 8. Endocrinopatía (adrenal, tiroidea, } \\
\text { hipofisiaria, gonadal, paratiroidea, } \\
\text { pancreática) }\end{array}$ \\
\hline & $\begin{array}{l}\text { 9. Cambios en la piel (hiperpigmentación, } \\
\text { hipertricosis, hemangiomas } \\
\text { glomeruloides, plétora, acrocianosis, } \\
\text { eritema o flushing, uñas pálidas) }\end{array}$ \\
\hline & 10. Papiledema \\
\hline & 11. Trombocitosis/policitemia \\
\hline \multirow{7}{*}{$\begin{array}{l}\text { Otros signos y } \\
\text { síntomas }\end{array}$} & Dedos hipocráticos \\
\hline & Pérdida de peso \\
\hline & Hiperhidrosis \\
\hline & $\begin{array}{l}\text { Hipertensión pulmonar/neumopatía } \\
\text { restrictiva }\end{array}$ \\
\hline & Diátesis trombótica \\
\hline & Diarrea \\
\hline & Niveles bajos de cianocobalamina (B12) \\
\hline
\end{tabular}

POEMS: polyneuropathy, organomegaly, endocrinopathy, monoclonal protein, skin changes; VEGF: vascular endotelial growth factor. Adaptada de Nakanishi, et al., $1984^{4}$.

enfocado en caracterizar su presentación clínica, paraclínica y en menor medida electrofisiológica. Existen muy pocos estudios que se enfoquen en evaluar la asociación potencial entre la presentación clínica y el patrón electrofisiológico (p. ej., desmielinizante frente al patrón axonal) del síndrome POEMS ${ }^{12,13}$. Por lo tanto, nos propusimos realizar un análisis sobre el patrón electrofisiológico y su correspondencia con la presentación clínica de una serie de pacientes con síndrome POEMS, para compararlo con la literatura previa similar. 


\section{Método}

En este estudio retrospectivo y observacional presentamos una descripción detallada de pacientes que acuden a atención médica al Departamento de Neurología y la Clínica de Enfermedades Neuromusculares del Instituto Nacional de Ciencias Médicas y Nutrición Salvador Zubirán, Ciudad de México. Se contó con la aprobación por el comité de investigación y por el comité de ética en investigación del instituto, con exención de la firma de un consentimiento bajo información, al tratarse de un estudio retrospectivo.

Todos los expedientes médicos con diagnóstico consignado de síndrome POEMS atendidos en el instituto en el periodo de tiempo de 2009 a 2019 fueron incluidos en una base de datos electrónica diseñada para la recolección de información clínica, laboratorio, imagen y electrofisiológica. Se excluyeron los casos con información faltante o en proceso de diagnóstico aunque incompleto, o aquellos en los que el estudio de electrodiagnóstico fuera realizado con una diferencia mayor a seis meses respecto al diagnóstico final del síndrome POEMS. En los pacientes con más de un estudio de electrodiagnóstico se consignó y analizó el que estuviese más próximo a la fecha de integración del diagnóstico final de POEMS. La exigencia de un estudio de electrodiagnóstico reciente al momento de la integración del POEMS obedece al objetivo general de evaluar la asociación de la presentación clínica y electrofisiológica del síndrome, con el fin de distinguir potenciales diferencias entre el patrón desmielinizante y el axonal. Se descartaron además los casos en los que no se cumplieran a cabalidad los criterios diagnósticos del síndrome POEMS, tal como se describe en las pautas clínicas vigentes ${ }^{3,10,20}$, así como los casos en los que existiera alguna otra explicación para la neuropatía, como diabetes mellitus, alcoholismo o uso de quimioterapia, entre otras etiologías. Aunque en casos excepcionales la polineuropatía puede preceder con muchos años al diagnóstico de POEMS ${ }^{17,18}$, finalmente también se excluyeron los casos en los que la polineuropatía precediera al diagnóstico de POEMS con $\geq 3$ años, con el fin de excluir otros diagnósticos diferenciales con mayor precisión, y que pudieran confundir los resultados de las evaluaciones de electrodiagnóstico en una muestra pequeña.

Para la recolección de los datos se solicitó al departamento de informática médica todos los expedientes con diagnósticos de síndrome POEMS. Luego se procedió a revisar para descartar aquellos que no cumplieran con los criterios de selección. Los datos de los expedientes seleccionados fueron revisados exclusivamente por el autor del trabajo, el cual utilizó una hoja de captura de datos para extraer la información necesaria para el estudio. En los formularios de recolección se utilizó un método de codificación que solo fue conocido por el autor, para proteger la confidencialidad de estos.

Se empleó el Medical Research Council (MRC) sum score como una variable cuantitativa que expresara la severidad de la afección motora, en la que 0 expresa el peor estado (plejía total) y 60 puntos el mejor (estado de fuerza normal, o $5 / 5$ en todos los segmentos evaluados). EI MRC sum score fue comparado entre los patrones electrofisiológicos axonal, desmielinizante y mixto.

Todas las operaciones estadísticas fueron realizadas con el programa informático para análisis de datos SPSS versión 20.0 para iOS (IBM Inc., EE.UU.). Con la información recolectada se procedió a presentar los datos mediante tablas y se analizaron los resultados con medidas de tendencia central. Los datos demográficos se presentaron como frecuencias relativas simples en la forma de porcentajes. Las variables cuantitativas continuas de distribución normal se analizaron como promedios y desviación estándar, mientras que las de distribución no paramétrica se presentaron como medianas con límites mínimo y máximo, o con rangos intercuartilares, según sea el caso. Para el contraste de la hipótesis primaria se emplearon pruebas de comparación de medias para dos o más grupos, como sigue: para variables cuantitativas de distribución normal entre dos grupos se empleó la prueba t de Student; para variables cuantitativas de distribución normal entre más de dos grupos se empleó ANOVA de una sola vía. La prueba U de Mann-Whitney se empleó en la comparación de medianas, para variables continuas no paramétricas entre dos grupos, y la prueba de Kruskal-Wallis cuando se comparó a más de dos grupos. Todos los análisis fueron bilaterales y considerados significativos cuando $p<0.05$. Las tablas que se construyen con los análisis de los datos se contrastan con las tres publicaciones más grandes que presentan la información clínica y electrofisiológica de una manera comparable a la que se hace en el presente estudio, si bien no son las únicas series de casos, ni las más extensas ${ }^{5,11-16}$.

\section{Resultados}

Inicialmente se obtuvieron 33 expedientes clínicos de pacientes con diagnóstico de polineuropatía 
asociada a gammapatía monoclonal para su revisión. Tras excluir 12 expedientes, ya sea por datos incompletos o abordajes de diagnóstico en progreso $(n=4)$, por tratarse de un diagnóstico distinto a POEMS $(n=3)$, por tener otras causas para el daño en el nervio periférico además del diagnóstico de POEMS $(n=3)$ o por existir una neuropatía que precedió en más de tres años el diagnóstico de POEMS $(n=2)$, se analizaron finalmente 11 pacientes (8 varones) para el análisis de la potencial asociación clínico-electrofisiológica (Tabla 2). La mediana de la edad al momento del diagnóstico fue de 40 años (rango: 31-51, media: $37.19 \pm 15.67$ años). La mediana de la edad al inicio de los síntomas fue de 38 años (rango: 32-51, media: $38.0 \pm 16.19$ años). Así, el tiempo promedio entre el inicio de las manifestaciones clínicas y el diagnóstico de POEMS fue de $9.7 \pm 8.37$ meses. En los 11 casos la manifestación clínica inicial fue la polineuropatía. La mayoría de los pacientes presentaron un patrón axonal $(n=5)$, seguido del desmielinizante $(n=4)$ y mixto $(n=2)$. Debido a nuestros criterios de selección, la discrasia de células plasmáticas se observó en los 11 pacientes (6 casos con gammapatía $\lambda$ y 5 con $\kappa$, inmunoglobulina [Ig] G: $72 \%$, IgA: $18 \%$, IgM: $9 \%$ ), organomegalia en 8 , endocrinopatía en 6 , alteraciones cutáneas en 8 , sobrecarga de volumen en 8 , lesiones óseas en 7 , papiledema en 3 e hipertensión pulmonar en 2 casos.

Los 11 pacientes analizados presentaban debilidad al momento del diagnóstico de inicio distal y ascendente crónica en los miembros inferiores, con marcha equina en grados variables. Al diagnóstico, 5 pacientes presentaban también debilidad distal de miembros superiores. No obstante, las alteraciones sensitivas precedieron a las motoras en 6 casos ( 3 con alteraciones positivas y 3 con alteraciones sensitivas negativas). La arreflexia de miembros inferiores se encontró en los 11 pacientes analizados, con hiporreflexia de extremidades superiores en 7 casos y normorreflexia en 4. El MRC sum score fue menor entre los pacientes con el patrón axonal (mediana: 37.00, rango: 21-39, media: $31.40 \pm 8.65$ años), en comparación con el grupo desmielinizante (mediana: 49.5, rango: 44-52, media: $48.75 \pm 3.95$ años) o mixto (mediana: 36.00, rango: $34-38$, media: $36.00 \pm 2.82$ años). La mediana del MRC sum score fue significativamente menor en el grupo de pacientes con el patrón axonal en comparación con el resto (37.00, rango: 21 -39 vs. 45.5, rango: 34-52, respectivamente; $p=0.024$ ).

No se evocó amplitud motora en los estímulos de 22 nervios motores, todos de los miembros inferiores, y se encontró disminuida en el $59 \%$ de las amplitudes restantes analizadas (Tabla 3). La velocidad de conducción nerviosa se encontró sin respuesta en 34 de las ocasiones, disminuida en el $79 \%$ de la velocidad normal, con parecida distribución entre miembros superiores e inferiores, en un rango de $22-100 \mathrm{~m} / \mathrm{s}$, con una media porcentual de enlentecimiento del $93 \%$. Las latencias motoras estuvieron prolongadas más severamente en nervios ulnares que en nervios medianos motores y más afectados en tibiales que en nervios peroneos. Interesantemente, se encontraron más prolongadas las latencias en los miembros superiores que en los inferiores. No se encontraron bloqueos de la conducción.

El promedio de amplitudes de nervios sensitivos en miembros superiores fue normal, aunque a nivel del nervio sural se encontró una disminución promedio del $52 \%$ de la amplitud. La velocidad de conducción nerviosa sensitiva con mayor disminución en miembros superiores que inferiores, con promedio del $83 \%$ respecto a la normalidad, sin diferencia entre el mediano y el ulnar. La latencia de nervios sensitivos estuvo prolongada en el $225 \%$ en promedio, con una alteración mayor en miembros inferiores que en los superiores.

El tiempo promedio entre el inicio de los síntomas $y$ el diagnostico de POEMS fue distinto entre las patrones desmielinizante (16.29 \pm 13.04 meses), axonal $(10.53 \pm 4.92$ meses $)$ y mixto $(8.0 \pm 4.24$ meses $)$ $(p=0.45)$. No se encontraron diferencias estadísticamente significativas entre los patrones desmielinizante y axonal/mixto (16.29 vs. 9.81, respectivamente; $p=0.21$ ), ni entre los patrones axonal y desmielinizante/mixto (10.53 vs. 13.52, respectivamente; $p=0.21$ ). Tampoco se encontraron diferencias estadísticamente significativas en el número promedio de criterios de diagnóstico cumplidos (mayores, menores y asociados/otros) entre lo patrones axonal (11.6), desmielinizante (17.25) o mixto (14.0) $(p=0.27)$.

\section{Discusión}

En este estudio encontramos características muy similares a las informadas en otros estudios, lo que incluye a las series multicéntricas con mayor número de pacientes ${ }^{5,7,8,11-16}$. Sin embargo, con las limitaciones de un tamaño de muestra pequeño, no encontramos que los patrones electrofisiológicos difirieran en cuanto a la latencia del tiempo entre el inicio de las manifestaciones clínicas y el diagnóstico final del síndrome POEMS, ni en la carga de criterios 
Gaceta Médica de México. 2021;157

Tabla 2. Características de los pacientes con síndrome POEMS

\begin{tabular}{|c|c|c|c|c|}
\hline Características clínicas & $\begin{array}{l}\text { Estudio actual } \\
\qquad(n=11)\end{array}$ & $\begin{array}{l}\text { Nakanishi, et al., } 1984^{4} \\
(n=102)\end{array}$ & $\begin{array}{l}\text { Dispenzieri, et al., } 2003^{5} \\
\qquad(\mathrm{n}=99)\end{array}$ & $\begin{array}{l}\text { Li, et al., 20116 } \\
\quad(n=99)\end{array}$ \\
\hline Mediana de la edad en años (RIC) al diagnóstico & 43 & 46 & 51 & 45 \\
\hline Sexo masculino & $9(81)$ & $67(67)$ & $62(62)$ & $58(58)$ \\
\hline Polineuropatía, n (\%) & $11(100)$ & $102(100)$ & $99(100)$ & $98(99)$ \\
\hline Axonal & $5(45)$ & - & - & - \\
\hline Desmielinizante & $4(36)$ & - & - & - \\
\hline Mixta & $2(18)$ & - & - & - \\
\hline Organomegalia, $n(\%)$ & $8(72)$ & - & $50(50)$ & $85(86)$ \\
\hline Hepatomegalia & $3(27)$ & $80(82)$ & $24(24)$ & $47(47)$ \\
\hline Esplenomegalia & $3(27)$ & $36(39)$ & $22(22)$ & $70(71)$ \\
\hline Linfadenopatía & $7(63)$ & $62(65)$ & $26(26)$ & $74(75)$ \\
\hline Enfermedad de Castleman & $3(27)$ & $19(63)$ & $11(73)$ & $24(24)$ \\
\hline Endocrinopatía, n (\%) & $11(100)$ & - & - & - \\
\hline Eje gonadal & $6(54)$ & - & - & - \\
\hline Eje adrenal & $1(9)$ & - & - & - \\
\hline Prolactina aumentada & $3(27)$ & - & - & - \\
\hline Ginecomastia o galactorrea & 0 & - & - & - \\
\hline Diabetes mellitus & $3(27)$ & - & - & - \\
\hline Hipotiroidismo & $6(54)$ & - & $14(14)$ & $42(42)$ \\
\hline Pico monoclonal, n (\%) & $11(100)$ & - & $99(100)$ & $99(100)$ \\
\hline $\lg A-\lambda$ & $1(9)$ & $29(41)$ & $44(52)$ & $65(71)$ \\
\hline $\lg G-\lambda$ & $5(45)$ & $38(54)$ & $40(48)$ & $20(22)$ \\
\hline $\lg M-\lambda$ & 0 & 0 & $1(1)$ & 0 \\
\hline $\lg A-\kappa$ & $1(9)$ & $3(4)$ & 0 & $1(1)$ \\
\hline $\lg G-\kappa$ & $3(27)$ & $1(1)$ & 0 & $2(2)$ \\
\hline $\lg M-\kappa$ & $1(9)$ & 0 & 0 & 0 \\
\hline Cambios en la piel, n (\%) & $8(72)$ & - & $67(68)$ & $87(88)$ \\
\hline Hiperpigmentación & $2(9)$ & - & - & - \\
\hline Acrocianosis y plétora & 0 & - & - & - \\
\hline Hemangioma/telangiectasia & $3(27)$ & - & - & - \\
\hline Hipertricosis & $1(9)$ & - & - & - \\
\hline Engrosamiento & $2(18)$ & - & - & - \\
\hline Otros, n (\%) & $11(100)$ & - & - & - \\
\hline Papiledema & $3(27)$ & $56(62)$ & $29(29)$ & $56(64)$ \\
\hline Sobrecarga de volumen & $8(72)$ & - & $29(29)$ & $87(88)$ \\
\hline Edema periférico & $5(45)$ & $91(91)$ & $24(24)$ & $84(85)$ \\
\hline Ascitis & $5(45)$ & $53(62)$ & $7(7)$ & $54(55)$ \\
\hline Derrame pleural & $6(54)$ & $36(40)$ & $3(3)$ & $43(43)$ \\
\hline Lesiones óseas & $7(63)$ & $27(27)$ & $56(50)$ & $7(63)$ \\
\hline Osteoesclerótica & $5(45)$ & $19(19)$ & $31(55)$ & $5(45)$ \\
\hline Lítica & 0 & $8(8)$ & $8(14)$ & - \\
\hline Mixta & $2(18)$ & 0 & $17(30)$ & $2(18)$ \\
\hline Hipertensión pulmonar* & $2(18)$ & - & $5(5)$ & $36(36)$ \\
\hline Trombocitosis $^{\dagger}$ & $1(9)$ & - & $53(54)$ & $54(55)$ \\
\hline Policitemia $^{\ddagger}$ & $1(9)$ & - & $18(18)$ & $9(9)$ \\
\hline
\end{tabular}


Tabla 3. Comparación de las características neurofisiológicas de los pacientes con síndrome POEMS ( $=11)$

\begin{tabular}{|c|c|c|c|c|c|c|}
\hline \multirow[t]{2}{*}{ Características electrofisiológicas } & \multicolumn{3}{|c|}{ Estudio actual } & \multicolumn{3}{|c|}{ Ju-Hong, et al., $2005^{13}$} \\
\hline & $n$ & Media \pm DE distal & Media \pm DE (\%) & n & Media \pm DE distal & Media \pm DE $(\%)$ \\
\hline $\begin{array}{l}\text { Latencia motora (ms) } \\
\text { Mediano } \\
\text { Ulnar } \\
\text { Tibial } \\
\text { Peroneo }\end{array}$ & $\begin{array}{l}20 \\
22 \\
12 \\
10\end{array}$ & $\begin{array}{l}4.62 \pm 1.18 \\
5.30 \pm 4.50 \\
4.45 \pm 2.10 \\
4.28 \pm 0.79\end{array}$ & $\begin{array}{c}121.44 \pm 31 \\
139.47 \pm 118.40 \\
94.70 \pm 44.70 \\
91.10 \pm 16.80\end{array}$ & $\begin{array}{c}12 \\
12 \\
6 \\
10\end{array}$ & $\begin{array}{l}5.1 \pm 1.24 \\
3.73 \pm 1.11 \\
6.78 \pm 3.29 \\
4.99 \pm 1.06\end{array}$ & $\begin{array}{l}146.97 \pm 35.66 \\
141.41 \pm 42.13 \\
126.55 \pm 61.43 \\
101.84 \pm 21.60\end{array}$ \\
\hline $\begin{array}{l}\text { VCN motora }(\mathrm{m} / \mathrm{s}) \\
\text { Mediano } \\
\text { Ulnar } \\
\text { Tibial } \\
\text { Peroneo }\end{array}$ & $\begin{array}{l}20 \\
22 \\
12 \\
10\end{array}$ & $\begin{array}{c}40.55 \pm 9.50 \\
42.50 \pm 14.90 \\
37.20 \pm 5.90 \\
49.40 \pm 20.7\end{array}$ & $\begin{array}{c}82.60 \pm 19.30 \\
86.70 \pm 30.00 \\
84.60 \pm 13.40 \\
120.40 \pm 50.50\end{array}$ & $\begin{array}{c}12 \\
12 \\
6 \\
10\end{array}$ & $\begin{array}{c}32.82 \pm 8.97 \\
32.84 \pm 10.52 \\
29.63 \pm 11.71 \\
29.49 \pm 8.49\end{array}$ & $\begin{array}{l}60.63 \pm 16.57 \\
61.07 \pm 19.57 \\
78.42 \pm 30.98 \\
67.97 \pm 19.56\end{array}$ \\
\hline $\begin{array}{l}\text { CMAP }(\mathrm{mV}) \\
\text { Mediano } \\
\text { Ulnar } \\
\text { Tibial } \\
\text { Peroneo }\end{array}$ & $\begin{array}{l}22 \\
22 \\
12 \\
10\end{array}$ & $\begin{array}{l}4.40 \pm 1.87 \\
4.70 \pm 2.80 \\
5.10 \pm 2.70 \\
3.52 \pm 0.71\end{array}$ & $\begin{array}{l}- \\
- \\
- \\
-\end{array}$ & $\begin{array}{c}12 \\
12 \\
6 \\
10\end{array}$ & $\begin{array}{c}5.84 \pm 3.74 \\
6.68 \pm 3.1 \\
5.18 \pm 6.54 \\
1.38 \pm 1.97\end{array}$ & $\begin{array}{l}- \\
- \\
-\end{array}$ \\
\hline $\begin{array}{l}\text { SNAP }(m V) \\
\text { Mediano } \\
\text { Ulnar } \\
\text { Sural }\end{array}$ & $\begin{array}{l}21 \\
20 \\
10\end{array}$ & $\begin{array}{c}15.09 \pm 11.56 \\
16.65 \pm 9.65 \\
6.80 \pm 3.98\end{array}$ & $\begin{array}{l}- \\
- \\
-\end{array}$ & $\begin{array}{l}8 \\
9 \\
8\end{array}$ & $\begin{array}{c}9.9 \pm 6.31 \\
10.29 \pm 2.81 \\
9.69 \pm 6.8\end{array}$ & $\begin{array}{l}- \\
- \\
-\end{array}$ \\
\hline $\begin{array}{l}\text { VCN sensitivo }(\mathrm{m} / \mathrm{s}) \\
\text { Mediano } \\
\text { Ulnar } \\
\text { Sural }\end{array}$ & $\begin{array}{l}21 \\
20 \\
12\end{array}$ & $\begin{array}{c}39.09 \pm 9.77 \\
39.35 \pm 11.57 \\
38.70 \pm 13.90\end{array}$ & $\begin{array}{l}78.18 \pm 19.55 \\
78.70 \pm 23.14 \\
94.39 \pm 34.87\end{array}$ & $\begin{array}{l}8 \\
9 \\
8\end{array}$ & $\begin{array}{l}34.83 \pm 5.09 \\
33.93 \pm 5.05 \\
32.09 \pm 3.39\end{array}$ & $\begin{array}{c}98.82 \pm 14.45 \\
87.57 \pm 13.03 \\
93.52 \pm 9.89\end{array}$ \\
\hline $\begin{array}{l}\text { Latencia sensitiva (ms) } \\
\text { Mediano } \\
\text { Ulnar } \\
\text { Sural }\end{array}$ & $\begin{array}{l}20 \\
20 \\
10\end{array}$ & $\begin{array}{c}7.50 \pm 12.32 \\
5.21 \pm 5.41 \\
7.62 \pm 9.95\end{array}$ & $\begin{array}{l}- \\
- \\
-\end{array}$ & $\begin{array}{l}- \\
- \\
-\end{array}$ & $\begin{array}{l}- \\
- \\
-\end{array}$ & $\begin{array}{l}- \\
- \\
-\end{array}$ \\
\hline
\end{tabular}

CMAP: compound muscle action potential; DE: desviación estándar; POEMS: polyneuropathy, organomegaly, endocrinopathy, monoclonal protein, skin changes; SNAP: sensory nerve action potential; VCN: velocidad de conducción nerviosa.

diagnósticos o manifestaciones clínicas del síndrome. Esto es contrario a nuestra hipótesis de trabajo inicial, pues esperábamos que los pacientes con un patrón electrofisiológico axonal tuvieran una latencia más prolongada entre el inicio de los síntomas y el diagnóstico, y consecuentemente, un mayor número de criterios cumplidos. Esto sugiere que aunque los pacientes con síndrome POEMS y daño axonal presentan mayor discapacidad motora, esta no es dependiente del tiempo con la enfermedad o con la severidad de la implicación sistémica. Aunque este hallazgo puede resultar contraintuitivo, se ha planteado con anterioridad que la severidad de la presentación clínica puede estar más vinculada a la actividad de la enfermedad (la cual no es homogénea entre los pacientes) que al tiempo con esta o la magnitud de marcadores tales como el VEGF sérico o las concentraciones de citocinas ${ }^{19,20}$.

Al contrario del estudio de Ju-Hong, et al. ${ }^{13}$, se encontró mayor prolongación de latencias y disminución de las velocidades en las cuatro extremidades, así como mayor disminución de las amplitudes motoras, lo que es congruente con el patrón axonal y mixto, cuya representación fue ligeramente superior a lo esperado en nuestro estudio. La prolongación de las latencias estuvo más prolongada en nervios ulnares que medianos y más en tibiales que en peroneos, lo que discrepa en cómo lo informan Ju-Hong, et al. ${ }^{13}$. Como era de esperarse, no se encontraron bloqueos de la conducción, los cuales se describen más frecuentemente polineuropatías desmielinizantes inmunomediadas.

Además de la ya mencionada principal limitación que este estudio tiene en su tamaño de muestra pequeño, el diseño retrospectivo puede implicar que algunas variables de interés no hayan sido recogidas con la mayor exactitud que requería la hipótesis y objetivos del presente análisis. Tampoco evaluamos aquí las diferencias que los tres patrones electrofisiológicos pudieran presentar en la respuesta al tratamiento, o en las concentraciones de biomarcadores 
cuya importancia se ha subrayado en tiempos recientes, tales como interleucina (IL) 1, IL-6 o factor de necrosis tumoral alfa, entre otros ${ }^{1-3}$. Creemos, sin embargo, que estos resultados pueden ayudar en el diseño de estudios observacionales de mayor envergadura que contesten de forma definitiva si el patrón de electrofisiológico depende del tiempo con la enfermedad o de la actividad de esta, además de sus determinantes.

En conclusión, tomando como base los resultados actuales, creemos ahora que es poco probable que los patrones electrofisiológicos tengan una correspondencia clínica extraneurológica clara, sin embargo, esto deberá probarse de forma definitiva con un estudio prospectivo y longitudinal de mayor tamaño.

\section{Financiamiento}

Los autores de este artículo declaran que no existió ningún patrocinador o fuente de financiamiento para la ejecución de este estudio.

\section{Conflicto de intereses}

Los autores de este artículo declaran no tener ningún conflicto de intereses.

\section{Responsabilidades éticas}

Protección de personas y animales. Los autores declaran que para esta investigación no se han realizado experimentos en seres humanos ni en animales.

Confidencialidad de los datos. Los autores declaran que han seguido los protocolos de su centro de trabajo sobre la publicación de datos de pacientes.

Derecho a la privacidad y consentimiento informado. Los autores declaran que en este artículo no aparecen datos de pacientes.

\section{Bibliografía}

1. Keddie S, Lunn M. POEMS syndrome. Curr Opin Neurol. 2018:31:551-8.

2. Cerri F, Falzone Y, Riva N Quattrini A. An update on the diagnosis and management of the polyneuropathy of POEMS syndrome. J Neurol. 2019:266:258-67.

3. Dispenzieri A. POEMS syndrome: 2019 Update on diagnosis, risk-stratification, and management. Am J Hematol. 2019;94:812-27.

4. Nakanishi T, Sobue I, Toyokura Y. The Crow-Fukase syndrome: A study of 102 cases in Japan. Neurology. 1984;34:712-20.

5. Dispenzieri A, Kyle RA, Lacy MQ, Rajkumar SV, Therneau TM, Larson DR, et al. POEMS syndrome: definitions and long-term outcome. Blood. 2003;101:2496-506.

6. Li J, Zhou D, Huang Z, Jiao L, Duan M, Zhang W, et al. Clinical characteristics and long-term outcome of patients with POEMS syndrome in China. Ann Hematol. 2011:90:819-26.

7. Suichi T, Misawa S, Beppu M, Takahashi S, Sekiguchi Y, Shibuya K, et al. Prevalence, clinical profiles, and prognosis of POEMS syndrome in Japanese nationwide survey. Neurology. 2019;93:e975-e983.

8. Wang C, Huang XF, Cai QQ, Cao XX, Duan MH, Cai H. Prognostic study for overall survival in patients with newly diagnosed POEMS syndrome. Leukemia. 2017:31:100-6.

9. Michelle L, Mauermann M. The peripheral neuropathies of POEMS syndrome and Castleman disease. Hematol Oncol Clin N Am. 2018;32:153-63.

10. Join Task Force of the EFNS and the PNS. European Federation of Neurological Societies/Peripheral Nerve Society Guideline on management of paraproteinemic demyelinating neuropathies. Report of a join task force of the European Federation of Neurological Societies and the Peripheral Nerve Society. J Periph Nerv Sys. 2006;11:9-19.

11. Liu M, Zou Z, Guan Y, Li J, Zhou D, Cui L. Motor nerve conduction study and muscle strength in newly diagnosed POEMS syndrome. Muscle Nerve. 2015;51:19-23

12. Mauermann ML, Sorenson EJ, Dispenzieri A, Mandrekar J, Suarez GA, Dyck PJ, et al. Uniform demyelination and more severe axonal loss distinguish POEMS syndrome from CIDP. J Neurol Neurosurg Psychiatry. 2012;83:480-6.

13. Ju-Hong M, Yoon-Ho H, Kwang-Loo L. Electrophysiological features of patients with POEMS syndrome. Clin Neurophysiol. 2005;116:965-8.

14. Singh D, Wadhwa J, Kumar L, Raina V, Agarwal A, Kochupillai V. POEMS syndrome: experience with fourteen cases. Leuk Lymphoma. 2003:44:1749-52

15. Shi X, Hu S, Yu X, Zhuang Q, Luo M, Jiang Q, et al. Clinicopathologic analysis of POEMS syndrome and related diseases. Clin Lymphoma Myeloma Leuk. 2015;15:e1521.

16. Carmona-Ruiz HA, Rosales-Uvera SG, Ulloa-Córdoba IA, Orihuela-Rodríguez O, Santiago-Cerecedo E, González-Duarte A, et al. Left atrial function and volume by magnetic resonance in patients with hereditary amyloidosis. Rev Invest Clin. 2019;71:387-92.

17. Nguyen VH. POEMS Syndrome diagnosed 10 years after disabling peripheral neuropathy. Case Rep Med. 2011;2011:126209.

18. Plaza C, Arquero T, García-Raso A, Llamas P. Diagnosis of POEMS syndrome in a patient with long-standing neuropathy. Neurologia. 2019; 34:272-4.

19. Scarlato M, Previtali SC, Carpo M, Pareyson D, Briani C, Del Bo R, et al. Polyneuropathy in POEMS syndrome: role of angiogenic factors in the pathogenesis. Brain. 2005;128:1911-20.

20. Sung JY, Kuwabara S, Ogawara K, Kanai K, Hattori T. Patterns of nerve conduction abnormalities in POEMS syndrome. Muscle Nerve. 2002; 26:189-93. 